\title{
Chemical characterization of traditional varietal olive oils in East of Spain
}

\author{
I. López-Cortés a,*, D.C. Salazar-García ${ }^{\text {b,c }}$, B. Velázquez-Martí ${ }^{\text {d }}$, D.M. Salazar ${ }^{\text {a }}$ \\ a Departamento de Producción Vegetal, Universidad Politécnica de Valencia, Camino de Vera s/n, 46022 Valencia, Spain \\ ${ }^{\mathrm{b}}$ Research Group on Plant Foods in Hominin Dietary Ecology, Max-Planck Institute for Evolutionary Anthropology, Deutscher Platz 6, 04103 Leipzig, Germany \\ c Department of Human Evolution, Max-Planck Institute for Evolutionary Anthropology, Deutscher Platz 6, 04103 Leipzig, Germany \\ d Departamento de Ingeniería Rural y Agroalimentaria, Universidad Politécnica de Valencia, Camino de Vera s/n, 46022 Valencia, Spain
}

\section{A R T I C L E I N F O}

Article history:

Received 20 February 2013

Received in revised form 23 April 2013

Accepted 30 April 2013

\section{Keywords:}

Varietal differentiation

Fatty acids

Sterols

Tocopherols

\begin{abstract}
A B S T R A C T
The aim of this work has been to characterize the chemical composition of the eight most emblematic varietal olive oils from the West of the Mediterranean Sea. These were classified into two groups according to the International Olive Council (IOC norms): Sweet oils (Farga, Morruda and Serrana) which were compared with Arbequina as standard of the Spanish sweet oils; and bitter-spicy oils (Alfafara, Blanqueta, and Villalonga) that were compared with Picual, considered as the standard of the bitter-spicy olive oils. For the study, sampled trees were chosen in their geographically separated originating areas. They were cultivated in the traditional conditions. The variety of each sampled tree was previously identified by the International Union for the Protection of new Varieties for Plants (UPOV TG/99/4). We have attempted to find differences between these varieties based on their fatty acid and sterol components. Although our results of only the sterols in olive oils suggested that stigmasterol could allow the segregation of the varieties, our experience indicates that analysis of the main fatty acids (palmitic, oleic and linoleic), together with a PCA applied to all fatty acids and sterols, can be used to validate the varietal determinations with enough precision. In addition, $\alpha$-tocopherol can be used as differentiator in bitter-spicy oils.
\end{abstract}

(c) 2013 Elsevier Ltd. All rights reserved.

\section{Introduction}

Olive oil is one of the main components of the Mediterranean diet. The olive tree has been cultivated for thousands of years in this area. Many varietal oils can be classified as autochthones, however little is known about the chemical composition of many local oil varieties. They are highly appreciated all over the world for their delicious taste and aroma, as well as for their healthy and nutritional properties. These properties are mainly caused by their monounsaturated fatty acids (MUFA), polyunsaturated fatty acids (PUFA), and antioxidant components.

Several studies have attempted to find those variables that allow one to differentiate between oil varieties. The varietal differentiation is being socially requested by the protection systems of the high quality associated to autochthone olive oil varieties. Aparicio and Luna (2002) noted that sensory descriptors, physicochemical quality parameters (free acidity, peroxides, absorbance in the ultraviolet region), stability parameters (total phenols and oxidative stability index), and the amount of fatty acids cannot be employed to distinguish monovarietal

\footnotetext{
* Corresponding author. Tel.: + 34963879331.

E-mail address: islocor@upv.es (I. López-Cortés).
}

oils with similar chemical profiles. For this reason, many authors have instead used the fatty acid profile and other minor components to characterize oils in several areas of the world, including Portugal (Matos et al., 2006), Tunisia (Haddada et al., 2008; Zarrouk et al., 2008), certain areas of Spain (Pardo, Cuesta, Alvarruiz, Granell, \& Álvarez-Ortí, 2010), Jordan (Al Ismail, Ahmad, \& Al-Dabbas, 2011), and Greece (Longobardi et al., 2012). These studies have demonstrated that the content of these oils depends mainly on the olive tree variety (Mariani, Bellan, Lestini, \& Aparicio, 2006), although environmental conditions can also have influence in some chemical components.

A few studies have focused specifically on the oils from the Mediterranean coast of Spain, but these have used limited methods or samples. For example, Lerma-García, Concha-Herrera, Herrero-Martínez, and Simó-Alfonso (2009) did a first characterization of some olive oils from Comunidad Valenciana but focused on the sterol content, and did not consider the fatty acids or tocopherol. Concha-Herrera, LermaGarcía, Herrero-Martínez, and Simó-Alfonso (2009) used the statistical technique of principal component analysis based on three dimensions, but focused only on discriminating a small number of olive varieties. It is necessary to apply these chemical analyses to the most common cultivated varieties in this area. In this work, the traditional varietal olive oils most extended in the Western Mediterranean were analyzed to evaluate the varietal differentiation by means of their chemical components (fatty acids, sterols, and tocopherol). 


\section{Materials and methods}

\subsection{Sampling}

The varieties of local olive oils studied in this research were coming from the olive trees previously identified by the International Union for the Protection of new Varieties for Plants (UPOV guidelines TG/99/4) as cultivars of Alfafara, Blanqueta, Farga, Morruda, Serrana, and Villalonga. These are included in the Regulation of Protected Designation of Origin (POD) for Valencian Olive Oil. In addition, these varieties are the most cultivated in the east coast of Spain. These were classified into two groups according to the International Olive Council (IOC norms): Sweet oils (Farga, Morruda and Serrana) and bitter-spicy oils (Alfafara, Blanqueta, and Villalonga). All analyzed oils meet the standards of virgin extra olive oil, with acidity lower than $0.7^{\circ}$, peroxide index lower than $20 \mathrm{meq} / \mathrm{kg}$, $\mathrm{K} 270<0.22$ and $\mathrm{K} 232<2.5$.

Composition of sweet oils was compared with Arbequina oil obtained from trees with the same location, and the bitter-spicy oils were compared with the characteristics of Picual oils, also located in the Mediterranean coast. This was done because the oil composition of Arbequina and that of Picual are considered as a Spanish standard in each group, and are the most common cultivars in Spain. The sampled trees were located in traditional cultivation areas, without irrigation and similar handling. The soil was usually mobilized two or three times per year. The sampling was carried out following Frías, GarcíaOrtiz, Hermoso, Jiménez, and Uceda's (1991) recommendations. Plots with trees of the varieties studied were identified in different locations. Five trees of each variety were sampled, these trees were chosen from more than 100 trees of the same variety to be considered representative of each variety in each zone of clonal selection of material. Not all varieties existed at each site. For example, a tree Arbequina was evaluated in each sample area, but there are other varieties where some trees were evaluated in the same area, in the case of the Forge, five trees were in the Mastership, however two trees Blanqueta Channel were in Navarrés, and three were in the mountains of Alicante. Fig. 1 allows the distribution point trees. About $6 \mathrm{~kg}$ of olives for each tree was collected for three years (2009-2011). All fruits were collected in the same state of ripeness. The ripeness was measured by means of the colorimetric index, proposed by Centro Investigacion Formacion Agraria (CIFA, Jaen, Spain), reported by Vera (2011), which reached 3/3.5. An Abencor MC2 oil extractor system (Ingenieria y Sistemas S.L., Sevilla,
Spain) was used to draw out the olive paste. After milling, the olive paste was fed into the thermomixer for $30 \mathrm{~min}$ and subsequently centrifuged at $5000 \mathrm{rpm}$ for $5 \mathrm{~min}$, after which the olive oil was recovered.

\subsection{Fatty acid determination}

For fatty acid determination, olive oil was subjected to transesterification with methanolic potassium hydroxide and n-heptane. The n-heptane extract was used to separate the fatty acid methyl esters. For this, a Varian 3400 gas chromatographer (Varian Associates, Walnut Creek, California, USA) equipped with a Combi-Pal (CTC Analytics, Zwingen, Switzerland) autosampler and a flame ionization detector was used. The following fatty acids were determined: palmitic acid (C16:0), palmitoleic acid (C16:1), heptadecanoic acid (C17:0), stearic acid (C18:0), oleic acid (C18:1), linoleic acid (C18:2), linolenic acid (C18:3), eicosanoic-arachidic acid (C20:0), docosanoic-behemic acid (C22:0), and tetracosanoic-lignoceric acid (C24:0). Results for fatty acid content were expressed as percentage of the total fatty acid methyl esters present in the olive oil. From these determinations, the percentage of total saturated (SFA), monounsaturated (MUFA) and polyunsaturated (PUFA) fatty acids over the total fatty acid content were calculated.

\subsection{Sterol determination}

Three sterols were examined $\beta$-sitosterol, stigmasterol and campesterol. These were analyzed according to the procedures described by Sanchez Casas, Osorio Bueno, Montano Garcia, and Martinez Cano (2004) who modified the method of Slover, Thompson, and Merola (1983). The oil sample was saponified with ethanolic potassium hydroxide solution. The unsaponifiable fraction was removed with ethyl ether. The unsaponifiable sterol fraction was separated by silica gel plate chromatography. Separation and quantification of the silanized sterol fraction was carried out by means of capillary column in a gas chromatograph, Hewlett-Packard model HP 5840 gas chromatograph, equipped with an FID-300, which worked at $290{ }^{\circ} \mathrm{C}$. The sample was injected at $280{ }^{\circ} \mathrm{C}$, following an isothermal process at $265^{\circ} \mathrm{C}$ for $45 \mathrm{~min}$ using a HP-5MS capillary column $(30 \mathrm{~m} \times 0.25 \mathrm{~mm} \times 0.22 \mu \mathrm{m})$. This column was filled with film OB5 de Tracer-Tecnocroma. The working conditions were as follows: Helium flow was $1 \mathrm{~mL} / \mathrm{min}$; the injector temperature

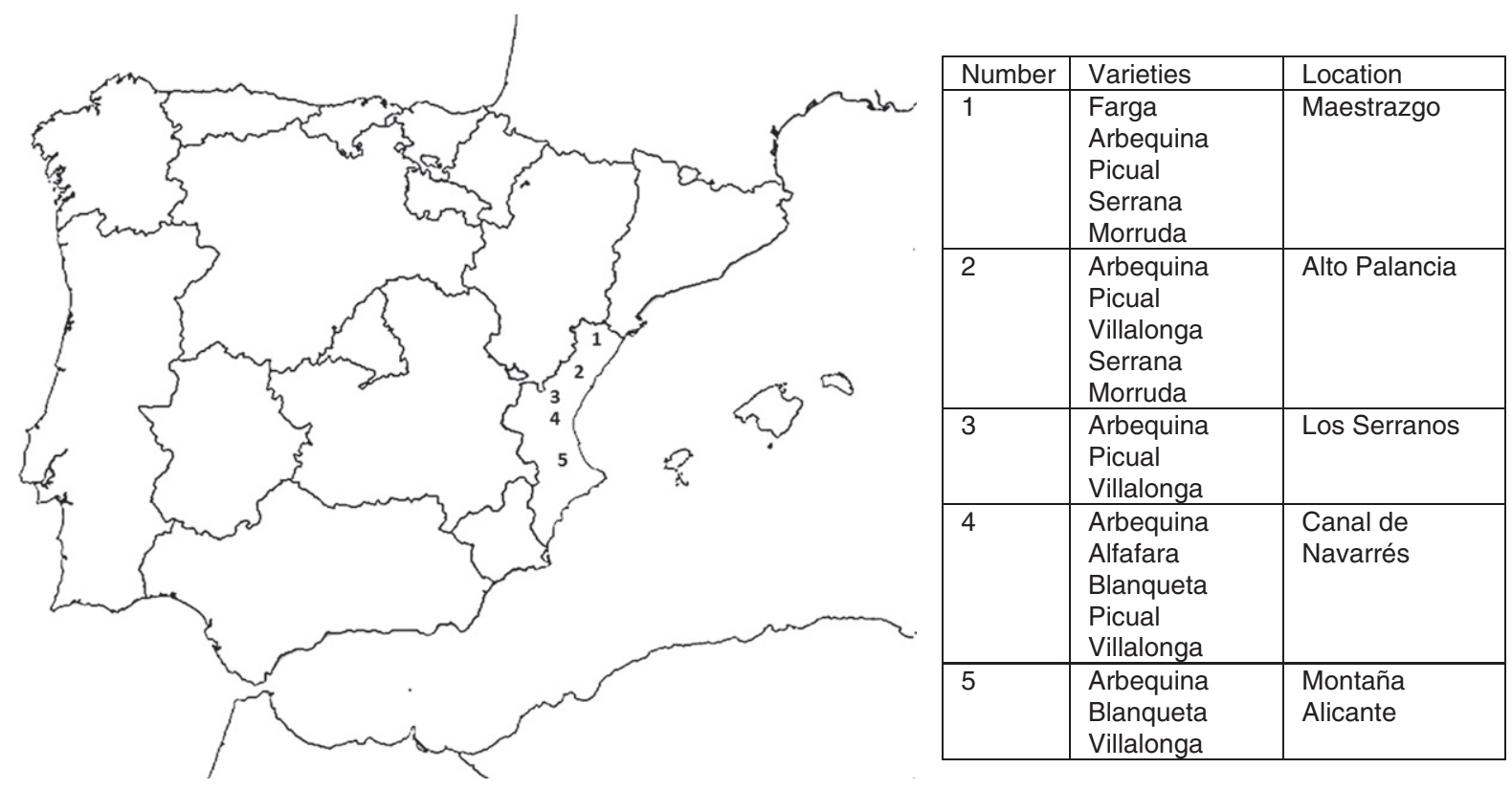

Fig. 1. Location of sampled cultivar to obtain the oil. 
Table 1

Mean and standard deviation of fatty acids of the different monovarietal olive oils of East of Spain.

\begin{tabular}{|c|c|c|c|c|c|c|c|c|c|c|c|c|c|}
\hline & $\begin{array}{l}\text { C16:0 } \\
\text { (palmitic ac) } \\
\%\end{array}$ & $\begin{array}{l}\text { C16:1 } \\
\text { (palmitoleic ac) } \\
\%\end{array}$ & $\begin{array}{l}\text { C17:0 } \\
\text { (heptadecanoic ac) } \\
\%\end{array}$ & $\begin{array}{l}\text { C18:0 } \\
\text { (stearic ac) } \\
\%\end{array}$ & $\begin{array}{l}\text { C18:1 } \\
\text { (oleic ac) } \\
\%\end{array}$ & $\begin{array}{l}\text { C18:2 } \\
\text { (linoleic ac) } \\
\%\end{array}$ & $\begin{array}{l}\text { C18:3 } \\
\text { (linolenic ac) } \\
\%\end{array}$ & $\begin{array}{l}\text { C20:0 } \\
\text { (eicosanoic ac) } \\
\%\end{array}$ & $\begin{array}{l}\text { C22:0 } \\
\text { (docosanoic ac) } \\
\%\end{array}$ & $\begin{array}{l}\text { C24:0 } \\
\text { (tetracosanoic ac) } \\
\%\end{array}$ & $\sum_{\%}^{\Sigma S F A s}$ & $\begin{array}{l}\sum \text { PUFAs } \\
\%\end{array}$ & $\sum_{\%}^{\sum M U F A s}$ \\
\hline \multicolumn{14}{|c|}{ Farga variety } \\
\hline $\mathrm{F} 1$ & $9.81 \pm 0.02$ & $0.63 \pm 0.02$ & $0.04 \pm 0.01$ & $2.00 \pm 0.02$ & $79.25 \pm 0.38$ & $7.95 \pm 0.07$ & $0.67 \pm 0.27$ & $0.33 \pm 0.01$ & $0.13 \pm 0.01$ & $0.04 \pm 0.01$ & 12.35 & 8.62 & 79.88 \\
\hline $\mathrm{F} 2$ & $9.68 \pm 0.04$ & $0.65 \pm 0.01$ & $0.03 \pm 0.01$ & $2.00 \pm 0.01$ & $79.17 \pm 0.46$ & $8.07 \pm 0.04$ & $0.49 \pm 0.01$ & $0.32 \pm 0.01$ & $0.13 \pm 0.01$ & $0.04 \pm 0.01$ & 12.20 & 8.56 & 79.82 \\
\hline F3 & $9.82 \pm 0.13$ & $0.65 \pm 0.02$ & $0.04 \pm 0.01$ & $2.00 \pm 0.01$ & $79.40 \pm 0.36$ & $7.80 \pm 0.16$ & $0.49 \pm 0.02$ & $0.32 \pm 0.01$ & $0.12 \pm 0.00$ & $0.04 \pm 0.01$ & 12.34 & 8.29 & 80.05 \\
\hline F4 & $9.98 \pm 0.03$ & $0.65 \pm 0.03$ & $0.03 \pm 0.01$ & $1.99 \pm 0.01$ & $79.51 \pm 0.20$ & $7.86 \pm 0.06$ & $0.50 \pm 0.01$ & $0.33 \pm 0.01$ & $0.13 \pm 0.00$ & $0.04 \pm 0.01$ & 12.50 & 8.36 & 80.16 \\
\hline F5 & $9.93 \pm 0.09$ & $0.66 \pm 0.01$ & $0.03 \pm 0.01$ & $1.99 \pm 0.02$ & $79.62 \pm 0.19$ & $8.00 \pm 0.02$ & $0.50 \pm 0.01$ & $0.33 \pm 0.00$ & $0.12 \pm 0.00$ & $0.04 \pm 0.01$ & 12.44 & 8.50 & 80.28 \\
\hline \multicolumn{14}{|c|}{ Morruda variety } \\
\hline M1 & $12.55 \pm 0.23$ & $0.69 \pm 0.02$ & $0.06 \pm 0.01$ & $2.63 \pm 0.02$ & $73.47 \pm 0.15$ & $11.13 \pm 0.24$ & $0.59 \pm 0.01$ & $0.40 \pm 0.01$ & $0.11 \pm 0.00$ & $0.04 \pm 0.01$ & 15.79 & 11.72 & 74.16 \\
\hline M2 & $12.86 \pm 0.05$ & $0.69 \pm 0.01$ & $0.07 \pm 0.01$ & $2.54 \pm 0.08$ & $72.85 \pm 0.19$ & $11.17 \pm 0.21$ & $0.60 \pm 0.01$ & $0.39 \pm 0.01$ & $0.12 \pm 0.01$ & $0.04 \pm 0.01$ & 16.02 & 11.77 & 73.54 \\
\hline M3 & $12.38 \pm 0.07$ & $0.70 \pm 0.01$ & $0.06 \pm 0.00$ & $2.58 \pm 0.03$ & $73.28 \pm 0.13$ & $8.39 \pm 6.14$ & $0.60 \pm 0.01$ & $0.40 \pm 0.01$ & $0.12 \pm 0.01$ & $0.04 \pm 0.01$ & 15.58 & 8.99 & 73.98 \\
\hline M4 & $12.40 \pm 0.26$ & $0.69 \pm 0.01$ & $0.06 \pm 0.00$ & $2.58 \pm 0.04$ & $74.20 \pm 0.22$ & $11.39 \pm 0.21$ & $0.62 \pm 0.01$ & $0.40 \pm 0.01$ & $0.11 \pm 0.01$ & $0.04 \pm 0.01$ & 15.59 & 12.01 & 74.89 \\
\hline M5 & $12.62 \pm 0.02$ & $0.60 \pm 0.01$ & $0.07 \pm 0.00$ & $2.55 \pm 0.07$ & $73.94 \pm 0.67$ & $11.48 \pm 0.33$ & $0.60 \pm 0.01$ & $0.41 \pm 0.01$ & $0.11 \pm 0.01$ & $0.04 \pm 0.00$ & 15.80 & 12.08 & 74.54 \\
\hline \multicolumn{14}{|c|}{ Serrana variety } \\
\hline S1 & $11.96 \pm 0.21$ & $0.74 \pm 0.11$ & $0.08 \pm 0.02$ & $2.16 \pm 0.10$ & $71.52 \pm 1.50$ & $13.79 \pm 2.08$ & $0.70 \pm 0.06$ & $0.39 \pm 0.03$ & $0.13 \pm 0.01$ & $0.05 \pm 0.01$ & 14.77 & 14.49 & 72.26 \\
\hline S2 & $11.31 \pm 0.73$ & $0.82 \pm 0.01$ & $0.07 \pm 0.01$ & $2.09 \pm 0.16$ & $72.96 \pm 0.24$ & $14.61 \pm 0.38$ & $0.64 \pm 0.01$ & $0.37 \pm 0.04$ & $0.13 \pm 0.01$ & $0.04 \pm 0.01$ & 14.01 & 15.25 & 73.78 \\
\hline S3 & $11.78 \pm 0.02$ & $0.72 \pm 0.03$ & $0.09 \pm 0.01$ & $2.09 \pm 0.01$ & $71.97 \pm 0.63$ & $14.64 \pm 0.19$ & $0.68 \pm 0.03$ & $0.38 \pm 0.01$ & $0.13 \pm 0.01$ & $0.05 \pm 0.01$ & 14.52 & 15.32 & 72.69 \\
\hline S4 & $11.70 \pm 0.07$ & $0.85 \pm 0.01$ & $0.08 \pm 0.01$ & $2.19 \pm 0.02$ & $72.66 \pm 0.19$ & $14.75 \pm 0.25$ & $0.72 \pm 0.01$ & $0.38 \pm 0.02$ & $0.14 \pm 0.01$ & $0.04 \pm 0.01$ & 14.53 & 15.47 & 73.51 \\
\hline S5 & $11.74 \pm 0.04$ & $0.87 \pm 0.02$ & $0.08 \pm 0.02$ & $2.12 \pm 0.02$ & $72.85 \pm 0.16$ & $15.10 \pm 0.02$ & $0.67 \pm 0.02$ & $0.37 \pm 0.05$ & $0.15 \pm 0.01$ & $0.05 \pm 0.01$ & 14.51 & 15.77 & 73.72 \\
\hline \multicolumn{14}{|c|}{ Arbequina variety } \\
\hline A1 & $15.64 \pm 0.05$ & $1.60 \pm 0.06$ & $0.12 \pm 0.01$ & $1.74 \pm 0.01$ & $78.55 \pm 0.60$ & $11.55 \pm 0.07$ & $0.59 \pm 0.05$ & $0.33 \pm 0.01$ & $0.10 \pm 0.01$ & $0.06 \pm 0.01$ & 17.99 & 12.14 & 80.15 \\
\hline A2 & $15.70 \pm 0.01$ & $1.62 \pm 0.05$ & $0.12 \pm 0.01$ & $1.73 \pm 0.01$ & $77.78 \pm 1.31$ & $11.97 \pm 0.25$ & $0.57 \pm 0.01$ & $0.34 \pm 0.01$ & $0.09 \pm 0.01$ & $0.05 \pm 0.01$ & 18.03 & 12.54 & 79.40 \\
\hline A3 & $15.63 \pm 0.04$ & $1.65 \pm 0.03$ & $0.12 \pm 0.01$ & $1.70 \pm 0.01$ & $79.36 \pm 0.59$ & $11.72 \pm 0.10$ & $0.57 \pm 0.01$ & $0.34 \pm 0.01$ & $0.09 \pm 0.01$ & $0.04 \pm 0.01$ & 17.92 & 12.24 & 81.01 \\
\hline A4 & $15.66 \pm 0.05$ & $1.60 \pm 0.03$ & $0.11 \pm 0.00$ & $1.75 \pm 0.00$ & $79.35 \pm 0.70$ & $11.90 \pm 0.02$ & $0.54 \pm 0.01$ & $.0 .34 \pm 0.00$ & $0.09 \pm .0 .00$ & $0.05 \pm 0.01$ & 18.00 & 12.44 & 80.95 \\
\hline A5 & $14.17 \pm 2.57$ & $1.66 \pm 0.03$ & $0.12 \pm 0.00$ & $1.73 \pm 0.01$ & $79.26 \pm 0.55$ & $12.00 \pm 0.01$ & $0.56 \pm 0.01$ & $0.34 \pm 0.01$ & $0.09 \pm 0.01$ & $0.05 \pm 0.01$ & 16.50 & 12.56 & 80.92 \\
\hline \multicolumn{14}{|c|}{ Alfafara variety } \\
\hline AL1 & $15.05 \pm 0.02$ & $1.23 \pm 0.01$ & $0.17 \pm 0.01$ & $1.73 \pm 0.01$ & $70.65 \pm 0.03$ & $9.41 \pm 0.02$ & $0.72 \pm 0.00$ & $0.36 \pm 0.00$ & $0.11 \pm 0.01$ & $0.05 \pm 0.01$ & 17.47 & 10.13 & 71.88 \\
\hline AL2 & $15.06 \pm 0.02$ & $1.26 \pm 0.01$ & $0.16 \pm 0.00$ & $1.79 \pm 0.01$ & $70.59 \pm 0.01$ & $9.45 \pm 0.01$ & $0.73 \pm 0.00$ & $0.34 \pm 0.00$ & $0.11 \pm 0.00$ & $0.06 \pm 0.01$ & 17.52 & 10.18 & 71.85 \\
\hline AL3 & $15.07 \pm 0.01$ & $1.26 \pm 0.00$ & $0.14 \pm 0.05$ & $1.78 \pm 0.04$ & $70.54 \pm 0.01$ & $9.48 \pm 0.08$ & $0.72 \pm 0.01$ & $0.34 \pm 0.03$ & $0.11 \pm 0.00$ & $0.05 \pm 0.01$ & 17.49 & 10.20 & 71.80 \\
\hline AL4 & $14.96 \pm 0.06$ & $1.29 \pm 0.03$ & $0.15 \pm 0.05$ & $1.74 \pm 0.02$ & $70.74 \pm 0.22$ & $9.61 \pm 0.01$ & $0.70 \pm 0.01$ & $0.34 \pm 0.00$ & $0.09 \pm 0.00$ & $0.05 \pm 0.01$ & 17.33 & 10.31 & 72.03 \\
\hline AL5 & $15.37 \pm 0.51$ & $1.32 \pm 0.04$ & $0.18 \pm 0.00$ & $1.75 \pm 0.01$ & $70.53 \pm 0.47$ & $9.47 \pm 0.01$ & $0.72 \pm 0.01$ & $0.34 \pm 0.01$ & $0.10 \pm 0.01$ & $0.05 \pm 0.01$ & 17.39 & 10.19 & 71.85 \\
\hline \multicolumn{14}{|c|}{ Blanqueta variety } \\
\hline B1 & $18.82 \pm 0.12$ & $1.70 \pm 0.12$ & $1.67 \pm 0.02$ & $1.93 \pm 0.05$ & $58.65 \pm 0.74$ & $16.64 \pm 0.06$ & $0.68 \pm 0.00$ & $0.37 \pm 0.03$ & $0.11 \pm 0.01$ & $0.05 \pm 0.01$ & 22.95 & 17.32 & 60.35 \\
\hline B2 & $18.56 \pm 0.13$ & $1.74 \pm 0.05$ & $0.16 \pm 0.04$ & $1.94 \pm 0.05$ & $59.56 \pm 1.05$ & $16.65 \pm 0.37$ & $0.69 \pm 0.02$ & $0.39 \pm 0.02$ & $0.11 \pm 0.01$ & $0.05 \pm 0.01$ & 21.21 & 17.34 & 61.30 \\
\hline B3 & $18.71 \pm 0.25$ & $1.72 \pm 0.04$ & $0.15 \pm 0.05$ & $1.95 \pm 0.06$ & $59.65 \pm 0.62$ & $16.66 \pm 0.27$ & $0.71 \pm 0.02$ & $0.38 \pm 0.00$ & $0.12 \pm 0.00$ & $0.05 \pm 0.01$ & 21.36 & 17.35 & 61.37 \\
\hline B4 & $17.30 \pm 0.40$ & $1.89 \pm 0.01$ & $0.10 \pm 0.01$ & $2.10 \pm 0.01$ & $58.35 \pm 0.78$ & $19.15 \pm 0.10$ & $0.80 \pm 0.01$ & $0.37 \pm 0.01$ & $0.13 \pm 0.00$ & $0.06 \pm 0.01$ & 20.06 & 19.95 & 60.24 \\
\hline B5 & $18.79 \pm 0.20$ & $1.76 \pm 0.03$ & $0.15 \pm 0.01$ & $1.99 \pm 0.03$ & $59.32 \pm 0.55$ & $16.59 \pm 0.18$ & $0.61 \pm 0.02$ & $0.38 \pm 0.01$ & $0.12 \pm 0.00$ & $0.05 \pm 0.01$ & 21.48 & 17.20 & 61.08 \\
\hline \multicolumn{14}{|c|}{ Villalonga variety } \\
\hline V1 & $14.38 \pm 0.37$ & $1.03 \pm 0.05$ & $0.13 \pm 0.01$ & $1.96 \pm 0.02$ & $68.50 \pm 0.62$ & $14.11 \pm 0.22$ & $0.88 \pm 0.01$ & $0.31 \pm 0.01$ & $0.09 \pm 0.00$ & $0.04 \pm 0.01$ & 16.91 & 14.99 & 69.53 \\
\hline V2 & $14.20 \pm 0.48$ & $1.10 \pm 0.02$ & $0.14 \pm 0.01$ & $1.94 \pm 0.02$ & $68.09 \pm 0.14$ & $14.18 \pm 0.22$ & $0.87 \pm 0.01$ & $0.30 \pm 0.01$ & $0.09 \pm 0.01$ & $0.04 \pm 0.01$ & 16.71 & 15.05 & 69.19 \\
\hline V3 & $14.67 \pm 0.12$ & $1.06 \pm 0.02$ & $0.13 \pm 0.01$ & $1.96 \pm 0.01$ & $67.86 \pm 0.35$ & $14.46 \pm 0.16$ & $0.87 \pm 0.02$ & $0.30 \pm 0.01$ & $0.10 \pm 0.01$ & $0.05 \pm 0.01$ & 17.21 & 15.33 & 68.92 \\
\hline V4 & $14.75 \pm 0.41$ & $1.02 \pm 0.01$ & $0.12 \pm 0.01$ & $1.96 \pm 0.01$ & $68.32 \pm 0.55$ & $14.39 \pm 0.20$ & $0.89 \pm 0.01$ & $0.31 \pm 0.01$ & $0.08 \pm 0.01$ & $0.05 \pm 0.01$ & 17.27 & 15.28 & 69.34 \\
\hline V5 & $14.49 \pm 0.42$ & $1.01 \pm 0.02$ & $0.13 \pm 0.01$ & $1.96 \pm 0.01$ & $68.12 \pm 0.17$ & $14.40 \pm 0.13$ & $0.92 \pm 0.01$ & $0.30 \pm 0.01$ & $0.09 \pm 0.01$ & $0.04 \pm 0.01$ & 17.01 & 15.32 & 69.13 \\
\hline \multicolumn{14}{|c|}{ Picual variety } \\
\hline P1 & $10.48 \pm 0.12$ & $0.64 \pm 0.01$ & $0.05 \pm 0.01$ & $2.94 \pm 0.01$ & $79.14 \pm 0.85$ & $5.41 \pm 0.34$ & $0.57 \pm 0.02$ & $0.33 \pm 0.01$ & $0.10 \pm 0.01$ & $0.04 \pm 0.01$ & 13.94 & 5.98 & 79.78 \\
\hline P2 & $10.75 \pm 0.24$ & $0.64 \pm 0.01$ & $0.05 \pm 0.01$ & $2.90 \pm 0.06$ & $79.24 \pm 0.35$ & $5.14 \pm 0.08$ & $0.58 \pm 0.01$ & $0.33 \pm 0.01$ & $0.11 \pm 0.01$ & $0.04 \pm 0.01$ & 14.18 & 5.72 & 79.88 \\
\hline P3 & $10.56 \pm 0.11$ & $0.63 \pm 0.01$ & $0.06 \pm 0.01$ & $2.90 \pm 0.01$ & $79.28 \pm 0.46$ & $5.17 \pm 0.06$ & $0.59 \pm 0.02$ & $0.34 \pm 0.01$ & $0.37 \pm 0.05$ & $0.04 \pm 0.01$ & 14.27 & 5.76 & 79.91 \\
\hline P4 & $10.47 \pm 0.11$ & $0.63 \pm 0.01$ & $0.05 \pm 0.01$ & $2.90 \pm 0.01$ & $79.87 \pm 0.21$ & $5.17 \pm 0.01$ & $0.57 \pm 0.01$ & $0.34 \pm 0.01$ & $0.10 \pm 0.01$ & $0.04 \pm 0.01$ & 13.90 & 5.74 & 80.50 \\
\hline P5 & $10.32 \pm 0.04$ & $0.65 \pm 0.01$ & $0.05 \pm 0.01$ & $2.89 \pm 0.01$ & $79.72 \pm 0.34$ & $5.14 \pm 0.01$ & $0.57 \pm 0.01$ & $0.34 \pm 0.01$ & $0.10 \pm 0.01$ & $0.04 \pm 0.01$ & 13.74 & 5.71 & 80.37 \\
\hline
\end{tabular}


was $300{ }^{\circ} \mathrm{C}$; and the detector temperature was $290{ }^{\circ} \mathrm{C}$. The injection volume was $0.2 \mathrm{~mL}$ at a flow rate of $1.1 \mathrm{~mL} / \mathrm{min}$ (Commission Regulation (EEC) No. 2568/91, corresponding to AOCS method Ch 6-91). The compounds were quantified by addition of an internal pattern (5- $\alpha$-cholestanol). The sterol concentration was expressed as $\mathrm{mg} / 100 \mathrm{~g}$ of fatty matter. The area of peaks generated by the sterols was carried out by an automatic integrator.

\section{4. $\alpha$-Tocopherol determination}

$\alpha$-Tocopherol was evaluated following AOCS method Ce 8-89. A solution of oil in hexane was analyzed on an Agilent Technologies HPLC system (1100 series) on a silica gel Lichrosorb Si-60 column (particle size $5 \mu \mathrm{m} \times 250 \mathrm{~mm} \times 4 \mathrm{~mm}$ i.d. of Sugerlabor, Madrid, Spain) using n-hexane/2-propanol $(98.5 / 1.5, \mathrm{vol} / \mathrm{vol})$ at a flow rate of $1 \mathrm{~mL} / \mathrm{min}$. A fluorescence detector (Thermo-Finnigan FL3000) was used, with excitation and emission wavelengths set at 290 and $330 \mathrm{~nm}$, respectively. The $\alpha$-tocopherol concentration was expressed as $\mathrm{mg} / \mathrm{kg}$ of fatty matter.

\subsection{Statistical analysis}

Data were subjected to analysis of variance (ANOVA) to test the effects of the variety in the means obtained from the three studied years for the each analyzed component. The locations where the trees were sampled had the same climate conditions and traditional cultivation systems, for this reason the location influences in minimum. On the other hand, because all varieties were not in all locations, multifactorial ANOVA is not possible. Subsequently, a principal component analysis (PCA) was performed because our design had one single categorical independent variable (variety) and multiple dependent variables (chemical parameters). This allows to standardize values using pairwise Euclidean distances among variety in the amount of fatty acids and sterols. Variables used in PCA were selected with regard to the ANOVA. Discriminant analysis was used to study the percentage of individual samples correctly classified according to the chemical component.

\section{Results and discussion}

Table 1 shows the averages and standard deviations of the ratios of fatty acids in the local and reference oils. Among the sweet olive oils the Farga variety had the highest content of the MUFAs, similar to the Arbequina variety, which is regularly used as standard in the composition in Spanish sweet olive oils. The lowest values were obtained in the Serrana and Morruda varieties, which did not differ significantly. The Serrana variety had the highest PUFAs, significantly larger than the Arbequina variety. Both groups are related to healthy oils (Madero et al., 2012; Saez, 2002). Among the local oils, Morruda had the highest SFA content, but this was still lower than Arbequina. These findings are in consonance with those reported by Tous and Romero (1993) for oils from an area further north than our study area.

With regard to MUFAs in the group of bitter-spicy varieties (Table 1), the Alfafara gave the highest values of three studied autochthone varieties but not as high as the reference variety (Picual), which was cultivated in the same areas. The MUFA content in Blanqueta was significantly lower than the other varieties, which is unsurprising, since previous studies have noted that this variety has a low oleic acid (C18:1) content (Tous \& Romero, 1993). It should be also noted that Blanqueta has a high linoleic acid (C18:2) content, which results in this variety having the highest PUFA content of the studied varieties. The PUFA content was also high in Villalonga and Alfafara, which was bigger than Picual. This was already reported by Tous and Romero (1993). Concerning SFA content, the Blanqueta oils have values exceptionally higher than all other studied oils

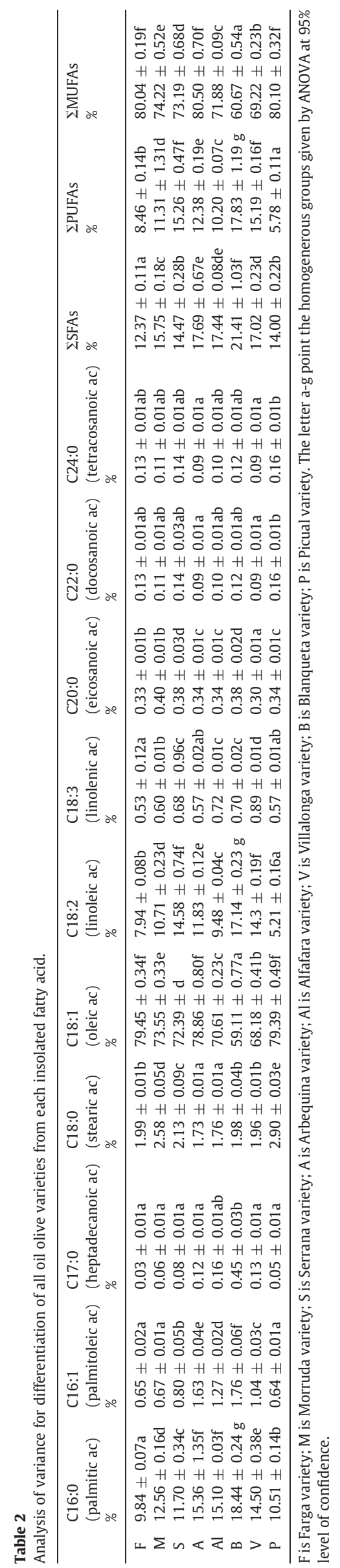


Table 3

Sterols and $\alpha$-tocopherol analyzed in the olive oil variety of East of Spain.

\begin{tabular}{|c|c|c|c|c|c|c|}
\hline & & & $\begin{array}{c}\beta \text {-Sitosterol } \\
\mathrm{mg} / 100 \mathrm{~g}\end{array}$ & $\begin{array}{c}\text { Campesterol } \\
\mathrm{mg} / 100 \mathrm{~g}\end{array}$ & $\begin{array}{l}\text { Stigmasterol } \\
\mathrm{mg} / 100 \mathrm{~g}\end{array}$ & $\begin{array}{c}\alpha \text {-Tocopherol } \\
\mathrm{mg} / \mathrm{kg}\end{array}$ \\
\hline \multirow[t]{20}{*}{ Sweet varieties } & \multirow[t]{5}{*}{ Farga } & $\mathrm{F} 1$ & $96.16 \pm 0.16$ & $2.97 \pm 0.02$ & $1.22 \pm 0.04$ & $264.42 \pm 4.84$ \\
\hline & & $\mathrm{F} 2$ & $98.67 \pm 0.47$ & $2.93 \pm 0.04$ & $1.17 \pm 0.06$ & $269.99 \pm 1.57$ \\
\hline & & F3 & $97.10 \pm 0.61$ & $2.96 \pm 0.03$ & $1.18 \pm 0.03$ & $267.57 \pm 4.86$ \\
\hline & & $\mathrm{F} 4$ & $97.67 \pm 0.48$ & $2.98 \pm 0.01$ & $1.15 \pm 0.03$ & $269.92 \pm 1.31$ \\
\hline & & F5 & $97.30 \pm 0.08$ & $2.95 \pm 0.01$ & $1.20 \pm 0.01$ & $269.89 \pm 0.40$ \\
\hline & \multirow[t]{5}{*}{ Morruda } & M1 & $96.72 \pm 0.57$ & $3.43 \pm 0.02$ & $1.69 \pm 0.04$ & $158.17 \pm 2.34$ \\
\hline & & M2 & $98.59 \pm 0.15$ & $3.40 \pm 0.02$ & $1.73 \pm 0.03$ & $158.41 \pm 0.99$ \\
\hline & & M3 & $98.68 \pm 0.06$ & $3.40 \pm 0.01$ & $1.71 \pm 0.05$ & $159.39 \pm 0.66$ \\
\hline & & M4 & $98.82 \pm 0.11$ & $3.43 \pm 0.02$ & $1.74 \pm 0.05$ & $159.70 \pm 0.51$ \\
\hline & & M5 & $98.38 \pm 0.34$ & $3.42 \pm 0.04$ & $1.78 \pm 0.02$ & $159.37 \pm 1.31$ \\
\hline & \multirow[t]{5}{*}{ Serrana } & $\mathrm{S} 1$ & $96.07 \pm 0.19$ & $2.94 \pm 0.05$ & $1.23 \pm 0.08$ & $184.53 \pm 2.92$ \\
\hline & & $\mathrm{S} 2$ & $96.73 \pm 0.78$ & $2.88 \pm 0.04$ & $1.26 \pm 0.03$ & $188.10 \pm 4.82$ \\
\hline & & S3 & $97.00 \pm 1.27$ & $2.98 \pm 0.03$ & $1.27 \pm 0.06$ & $192.43 \pm 2.29$ \\
\hline & & S4 & $97.15 \pm 0.48$ & $3.05 \pm 0.10$ & $1.23 \pm 0.05$ & $191.24 \pm 1.88$ \\
\hline & & S5 & $96.73 \pm 0.45$ & $3.12 \pm 0.01$ & $1.25 \pm 0.01$ & $192.41 \pm 2.21$ \\
\hline & \multirow[t]{5}{*}{ Arbequina } & $\mathrm{A} 1$ & $97.94 \pm 0.44$ & $2.87 \pm 0.02$ & $0.42 \pm 0.01$ & $190.66 \pm 7.42$ \\
\hline & & $\mathrm{A} 2$ & $98.20 \pm 0.38$ & $2.88 \pm 0.02$ & $0.44 \pm 0.01$ & $169.43 \pm 4.54$ \\
\hline & & A3 & $98.45 \pm 0.28$ & $2.89 \pm 0.01$ & $0.42 \pm 0.01$ & $192.23 \pm 2.51$ \\
\hline & & A4 & $98.91 \pm 0.81$ & $2.88 \pm 0.01$ & $0.44 \pm 0.01$ & $184.30 \pm 4.57$ \\
\hline & & A5 & $99.88 \pm 1.18$ & $2.88 \pm 0.01$ & $0.41 \pm 0.01$ & $189.65 \pm 1.30$ \\
\hline \multirow[t]{20}{*}{ Bitter-spicy varieties } & \multirow[t]{5}{*}{ Alfafara } & AL1 & $96.80 \pm 0.06$ & $2.95 \pm 0.01$ & $0.70 \pm 0.02$ & $215.43 \pm 4.25$ \\
\hline & & AL2 & $96.79 \pm 0.15$ & $2.97 \pm 0.01$ & $0.61 \pm 0.02$ & $211.68 \pm 2.34$ \\
\hline & & AL3 & $96.92 \pm 0.10$ & $2.93 \pm 0.01$ & $0.65 \pm 0.01$ & $215.35 \pm 3.54$ \\
\hline & & AL4 & $96.76 \pm 0.16$ & $2.96 \pm 0.03$ & $0.64 \pm 0.01$ & $218.39 \pm 1.17$ \\
\hline & & AL5 & $96.69 \pm 0.02$ & $2.96 \pm 0.02$ & $0.63 \pm 0.01$ & $219.03 \pm 0.86$ \\
\hline & \multirow[t]{5}{*}{ Blanqueta } & B1 & $95.53 \pm 0.45$ & $3.09 \pm 0.78$ & $0.88 \pm 0.09$ & $186.01 \pm 3.42$ \\
\hline & & B2 & $94.92 \pm 0.88$ & $3.51 \pm 0.03$ & $0.92 \pm 0.01$ & $187.59 \pm 1.59$ \\
\hline & & B3 & $96.17 \pm 0.65$ & $3.47 \pm 0.03$ & $0.59 \pm 0.03$ & $187.43 \pm 0.62$ \\
\hline & & B4 & $95.32 \pm 0.59$ & $3.40 \pm 0.01$ & $0.98 \pm 0.01$ & $181.21 \pm 0.99$ \\
\hline & & B5 & $95.62 \pm 0.70$ & $3.54 \pm 0.01$ & $0.95 \pm 0.02$ & $186.71 \pm 0.47$ \\
\hline & \multirow[t]{5}{*}{ Villalonga } & V1 & $93.88 \pm 1.98$ & $3.31 \pm 0.02$ & $1.99 \pm 0.02$ & $153.19 \pm 1.62$ \\
\hline & & V2 & $94.21 \pm 0.31$ & $3.32 \pm 0.01$ & $1.97 \pm 0.04$ & $151.52 \pm 1.53$ \\
\hline & & V3 & $94.69 \pm 0.40$ & $3.32 \pm 0.02$ & $1.98 \pm 0.02$ & $148.84 \pm 0.53$ \\
\hline & & V4 & $94.66 \pm 0.05$ & $3.33 \pm 0.03$ & $1.99 \pm 0.01$ & $149.88 \pm 0.66$ \\
\hline & & V5 & $94.51 \pm 0.12$ & $3.29 \pm 0.06$ & $1.96 \pm 0.03$ & $150.48 \pm 0.65$ \\
\hline & \multirow[t]{5}{*}{ Picual } & $\mathrm{P} 1$ & $96.68 \pm 0.57$ & $2.91 \pm 0.02$ & $0.35 \pm 0.01$ & $134.01 \pm 0.93$ \\
\hline & & $\mathrm{P} 2$ & $96.47 \pm 0.16$ & $2.93 \pm 0.01$ & $0.34 \pm 0.01$ & $134.85 \pm 0.54$ \\
\hline & & P3 & $96.34 \pm 0.24$ & $2.94 \pm 0.01$ & $0.32 \pm 0.01$ & $131.53 \pm 0.61$ \\
\hline & & $\mathrm{P} 4$ & $96.48 \pm 0.52$ & $2.93 \pm 0.01$ & $0.36 \pm 0.01$ & $138.11 \pm 0.42$ \\
\hline & & P5 & $96.61 \pm 0.22$ & $2.92 \pm 0.01$ & $0.36 \pm 0.01$ & $133.54 \pm 0.59$ \\
\hline
\end{tabular}

(bitter-spicy and sweet) because its content of palmitic acid is the highest. This contributes to the particularity of this oil variety, as has previously been reported by Iñiguez et al. (2001), and Civantos López-Villalta, Contreras, and Grana, (2008).

In Table 2 analysis of variance to compare all varieties are shown. The differences in the fatty acid composition were mainly remarkable for palmitic, oleic, and linoleic acids. Nevertheless, any ANOVA focused on only one fatty acid allowed the differentiation of all studied oils. For this reason in this research the combination of statistical techniques based on ANOVA and PCA is proposed.

Sterols are a very important group of compounds in the oils because they are related to the stability of the oil at high temperature. In addition, they are inhibitors of the polymerization reactions (Velasco \& Dobarganes, 2002). The sterols reach between of $20-23 \%$ of unsaponifiable fraction in olive oil. Gutierrez, Varona, and Albi (2000) and Lerma-García et al. (2009) noted that the sterol analysis allows to determine the authenticity and monovarietal origin of olive oils. Table 3 shows the means and standard deviation of the studied sterols in each variety. In addition, an ANOVA was done to evaluate if the varieties could be differentiated from one of some of these compounds (Table 3 ). Our results indicate that only the stigmasterol allows their segregation.

Among the tocopherols, we analyzed only the $\alpha$-tocopherol because it is the majority within this group of compounds (Aguilera et al., 2005; Beltran, Aguilera, Del Río, Sánchez, \& Martínez, 2005). The results showed that groups of oils both sweet and bitter-spicy under study can be clearly distinguished by $\alpha$-tocopherol content, except Arbequina and Serrana. The $\alpha$-tocopherol content showed highly significant differences $(p<0.05)$ among all cultivars (Table 3$)$. The ranges obtained in sweet olive oils were from $158.17 \mathrm{mg} / \mathrm{kg}$ in Morruda to $269.9 \mathrm{mg} / \mathrm{kg}$ in variety Farga. The three local bitter-spicy varieties gave higher values than the reference variety, Picual. The range was found from $131.53 \mathrm{mg} / \mathrm{kg}$ in Picual to $219.03 \mathrm{mg} / \mathrm{kg}$ in Alfafara.

Rightly, differences between the trees exist, which can be seen in Tables 1 and 3, but these differences cannot be associated to the location. Tables 2 and 4 show mean and differences between varieties. The interannual variations can be evaluated by the little values of standard deviation shown in Tables 1 and 3. If there were large differences between the years studied, the deviation would be great, but it's not.

Table 4

Analysis of variance of the sterols and $\alpha$-tocopherol in the studied varieties. The letter a-g point the homogenerous groups given by ANOVA at 95\% level of confidence.

\begin{tabular}{clllll}
\hline & & $\begin{array}{l}\beta-S i t o s t e r o l \\
\mathrm{mg} / 100 \mathrm{~g}\end{array}$ & $\begin{array}{l}\text { Campesterol } \\
\mathrm{mg} / 100 \mathrm{~g}\end{array}$ & $\begin{array}{l}\text { Stigmasterol } \\
\mathrm{mg} / 100 \mathrm{~g}\end{array}$ & $\begin{array}{l}\alpha \text {-Tocopherol } \\
\mathrm{mg} / \mathrm{kg}\end{array}$ \\
\hline \multirow{2}{*}{ Sweet } & Morruda & $98.23 \mathrm{bc}$ & $3.41 \mathrm{c}$ & $1.73 \mathrm{~d}$ & $159.01 \mathrm{a}$ \\
varieties & Arbequina & $98.68 \mathrm{c}$ & $2.88 \mathrm{a}$ & $0.43 \mathrm{a}$ & $185.25 \mathrm{~b}$ \\
& Serrana & $96.73 \mathrm{a}$ & $2.99 \mathrm{~b}$ & $1.25 \mathrm{c}$ & $189.74 \mathrm{~b}$ \\
& Farga & $97.38 \mathrm{ab}$ & $2.96 \mathrm{~b}$ & $1.18 \mathrm{~b}$ & $268.35 \mathrm{c}$ \\
Bitter- & Picual & $96.52 \mathrm{c}$ & $2.92 \mathrm{a}$ & $0.35 \mathrm{a}$ & $134.41 \mathrm{a}$ \\
spicy & Alfafara & $96.79 \mathrm{c}$ & $2.95 \mathrm{a}$ & $0.64 \mathrm{~b}$ & $215.98 \mathrm{~d}$ \\
varieties & Blanqueta & $95.51 \mathrm{~b}$ & $3.40 \mathrm{~b}$ & $0.86 \mathrm{c}$ & $185.79 \mathrm{c}$ \\
& Villalonga & $94.39 \mathrm{a}$ & $3.31 \mathrm{~b}$ & $1.98 \mathrm{~d}$ & $150.78 \mathrm{~b}$ \\
\hline
\end{tabular}



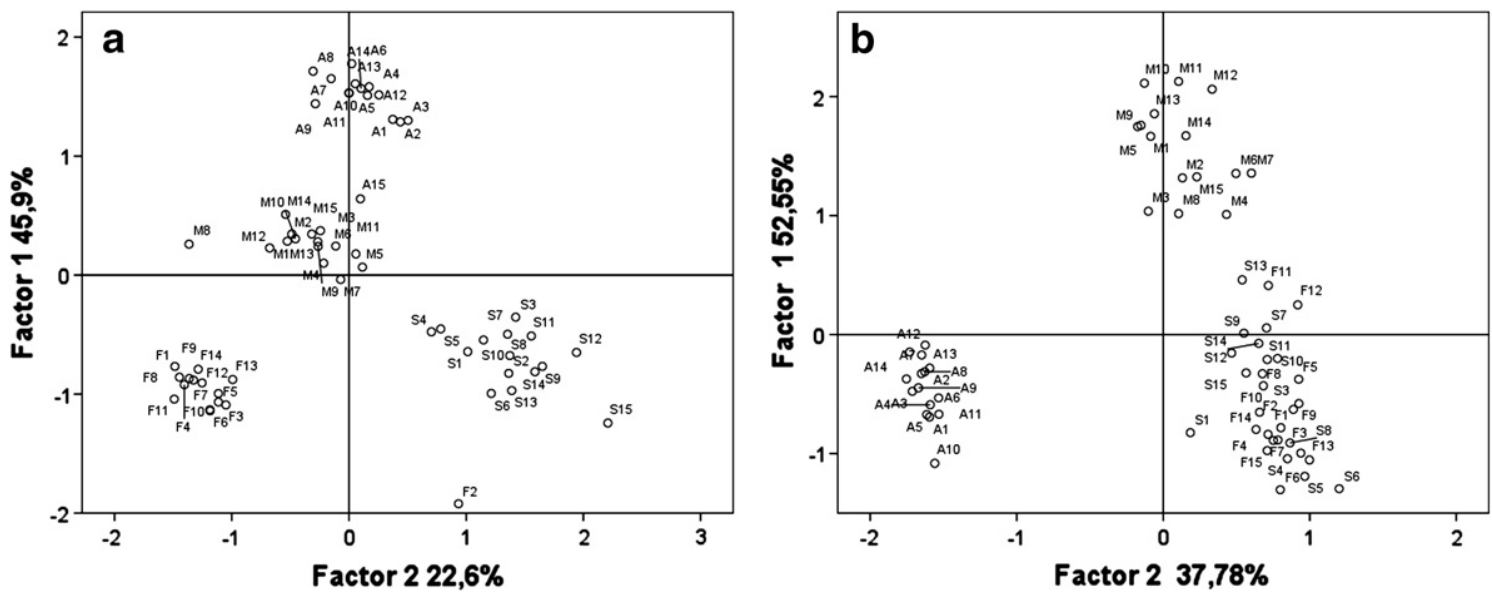

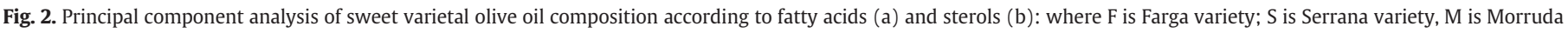
variety; and $\mathrm{A}$ is Arbequina variety.

Therefore, there were no significant differences between the oil obtained each year.

Fig. 2 illustrates the PCA results for the sweet oils, separating out the fatty acids studied (a) and sterols (b). In graph 1 a factors 1 and 2 explain the $45.90 \%$ and $22.60 \%$ of the variance of components of fatty acids in the samples, respectively. In graph $1 \mathrm{~b}$, factors 1 and 2 represent the $52.55 \%$ and $37.78 \%$ of the variance of sterol content. This means that more than $80 \%$ of the variability is explained in both kinds of components. According to these analyses a clear differentiation of the studied varietal olive oils according to fatty acids, which are located in specific areas on the graph can be pointed out. This allows applying thresholds to identify the oils. However, the varieties Farga and Serrana cannot be differentiated using only sterol analysis. This same finding was observed by Lerma-García et al. (2009).

The results of the PCA for fatty acid compounds for the bitterspicy oils are illustrated in Fig. 3. Like the sweet oils, these fatty acid profiles distinguish the oil varieties. However, the analysis of only the sterols does not clearly discriminate the olive oils of Picual, Alfafara and Blanqueta, because their cloud of points is very close. The two principal factors together explain $76.47 \%$ of the variance of fatty acids and $97.26 \%$ of the variance of sterols.

\section{Conclusions}

The characterization of autochthone monovarietal olive oils from the east coast of Spain has been well established with enough information. All the described results have shown a wide variability in the chemical characteristics of the studied virgin olive oils because of the diversity of the varieties, given that the sampled trees were cultivated with the same environmental and handling conditions. The differences in the fatty acid composition were mainly remarkable for palmitic, oleic and linoleic acids. Nevertheless, to discriminate the studied varieties it is necessary to combine a study of fatty acids with analyses of the sterols and $\alpha$-tocopherol, as well as by using both ANOVA and PCA.

The PCA of the sterols only allowed us to differentiate the sweet oil varieties except Serrana and Farga. An identical analysis of the bitter-spicy oils only clearly separates the Villalonga variety, nevertheless the other varieties show a closer data structure, but not mixed. Regarding $\alpha$-tocopherol, the results showed that groups of oils, both sweet and bitter-spicy under study, can be clearly differentiated except Arbequina and Serrana.

Though our results of only the sterols in olive oils suggested that stigmasterol could allow the segregation of the varieties, our experience indicates that analysis of the main fatty acids (palmitic, oleic and linoleic), together with a PCA applied to all fatty acids and sterols, can be used to carry out the varietal differentiation with enough precision. Combining the results obtained for the fatty acids and sterols in PCA analysis detects the influence of the variety in the oil chemical composition. In our opinion, at least in our working conditions, it is possible to differentiate the varieties studied, using this type of analysis. Therefore, they can be used for proper labeling and marketing of varietal oils.
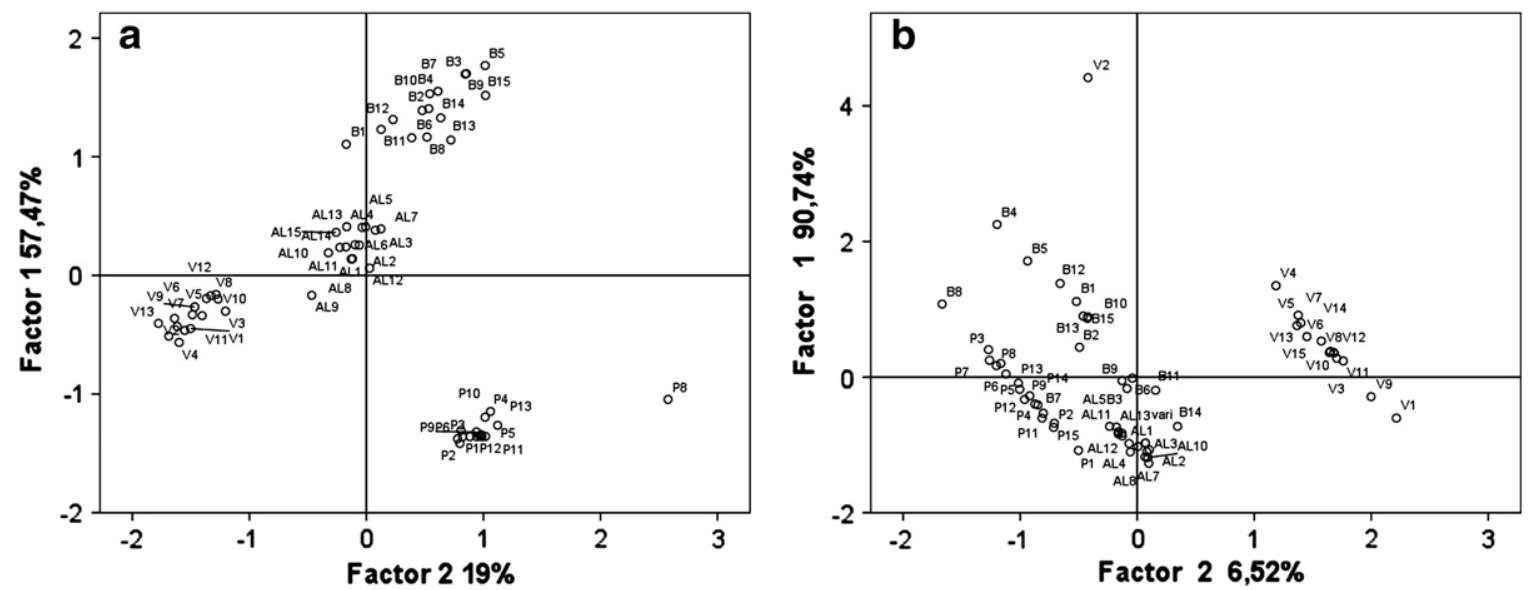

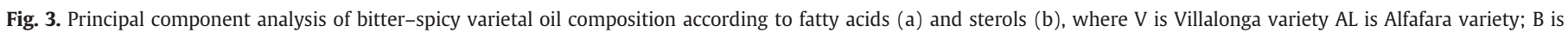
Blanqueta variety; and P is Picual Variety. 


\section{References}

Aguilera, M. P., Beltrán, G., Ortega, D., Fernández, A., Jiménez, A., \& Uceda, M. (2005). Characterisation of virgin olive of Italian olive cultivars "Frantoio" and "Leccino" grown in Analusia. Food Chemistry, 89, 387-391.

Al Ismail, K. M., Ahmad, R., \& Al-Dabbas, M. (2011). Some physiochemical properties of olive and olive oil of three Jordanian olive varieties. Rivista Italiana Delle Sostanze Grasse, 88(3), 191-198.

AOCS (1989). Determination of tocopherols and tocotrienols in vegetable oils and fats by HPLC. Official methods and recommended practices of the AOCS (4th ed.)USA: AOLS Press Champaign, IL (Official Method Ce 8-89).

Aparicio, R., \& Luna, G. (2002). Characterisation of monovarietal virgin olive oils. European Journal Lipid Science Technology, 104, 614-627.

Beltran, G., Aguilera, M. P., Del Río, C., Sánchez, S., \& Martínez, L. (2005). Influence of fruit ripening process on the natural antioxidant content of Hojiblanca virgin olive oils. Food Chemistry, 89, 207-215.

Civantos López-Villalta, L., Contreras, R., \& Grana, R. (2008). Obtención del aceite de oliva virgen. Madrid: Agrícola española (260 pp.).

Concha-Herrera, V., Lerma-García, M. J., Herrero-Martínez, J. M., \& Simó-Alfonso, E. F. (2009). Prediction of the genetic variety of extra virgin olive oils produced at La Comunitat Valenciana, Spain, by Fourier transform infrared spectroscopy. Journal of Agricultural and Food Chemistry, 57, 9985-9989.

European Union Commission Regulation 2568/91 (1991). Characteristics of olive and olive pomace oils and their analytical methods. Official Journal of the European Communitie, L248, 1-82.

Frías, L., García-Ortiz, A., Hermoso, M., Jiménez, A., \& Uceda, M. (1991). Analistas de laboratorio de almazara. Sevilla: Junta de Andalucía.

Gutierrez, F., Varona, I., \& Albi, M. A. (2000). Relation of acidity and sensory quality with sterol content of olive oil from stored fruit. Journal of Agricultural and Food Chemistry, 48, 1106-1110.

Haddada, F., Krichène, D., Manai, H., Oueslati, I., Daoud, D., \& Zarrouk, M. (2008). Analytical evaluation of six monovarietal virgin olive oils from Northern Tunisia. European Journal Lipid Science Technology, 110, 905-913.

Iñiguez, A., Paz, S., \& Illa, F. (2001). Variedades de olivo cultivadas en la Comunidad valenciana. Valencia: Generalitat Valenciana (267 pp.).

Lerma-García, M. J., Concha-Herrera, V., Herrero-Martínez, J. M., \& Simó-Alfonso, E. F. (2009). Classification of extra virgin olive oils produced at La Comunidad Valenciana according to their genetic variety using sterol profiles established by high performance liquid chromatography with mass spectrometry detection. Journal of Agricultural and Food Chemistry, 57(22), 10512-10517.

Longobardi, F., Ventrella, A., Casiello, G., Sacco, D., Tasioula-Margari, M., Kiritsakis, A. K., et al. (2012). Characterisation of the geographical origin of Western Greek virgin olive oils based on instrumental and multivariate statistical analysis. Food Chemistry, 133, 169-175.

Madero, A., Perri, E., Alessandrino, M., Ciliberti, A., Parise, A., \& Romano, E. (2012) Comparative study on the behavior of olive cultivars from different origins cultivated in the same environment, with regard to some important characteristics of the olive oil produced. VI International Symposium on Olive Growing, 949. (pp. 213-220): The Hague: International Society for Horticultural Science.

Mariani, C., Bellan, G., Lestini, E., \& Aparicio, R. (2006). The detection of the presence of hazelnut oil in olive oil by free and esterified sterols. European Food Resource Technology, 223, 655-661.

Matos, L., Cunha, S., Amaral, J., Pereira, J. A., Andrade, P., Seabra, R., et al. (2006). Chemometric characterization of three varietal olive oils (Cvs. Cobranc, osa Madural and Verdeal Transmontana) extracted from olives with different maturation indices. Food Chemistry, 102, 406-414.

Pardo, J. E., Cuesta, M. A., Alvarruiz, J. D., Granell, M., \& Álvarez-Ortí, M. (2010). Evaluation of potential and real qualities of virgin olive oil from the designation of origin (DO) "Aceite Montes de Alcaraz" (Albacete, Spain). Food Chemistry, 124 1684-1690.

Saez, G. (2002). Aceite de oliva y antioxidantes. El Aceite de oliva. Valencia: Fundación Valenciana de Estudios Avanzados.

Sanchez Casas, J., Osorio Bueno, E., Montano Garcia, A. M., \& Martinez Cano, M. (2004) Sterol and erythrodiol + uvaol content of virgin olive oils from cultivars of Extremadura (Spain). Food Chemistry, 87, 225-230.

Slover, H. T., Thompson, R. H., \& Merola, G. V. (1983). Determination of tocopherols and sterols by capillary gas chromatography. Journal of the American Chemical Society, $60,1524-1528$

Tous, J., \& Romero, A. (1993). Variedades del olivo. Barcelona: Fundación La Caixa. AEDOS (172 pp.).

Velasco, J., \& Dobarganes, C. (2002). Oxidative stability of virgin olive oil. European Journal of Lipid Science and Technology, 104, 661-676.

Vera, Hernández M. (2011). Aceite de oliva virgen extra su obtención y conservación. Madrid: AMV Ediciones (302 pp.).

Zarrouk, W., Faouzia, H., Baccouri, B., Oueslati, I., Taamalli, W., Fernandez, X., et al (2008). Characterization of virgin olive oil from Southern Tunisia. European Journal of Lipid Science and Technology, 110, 81-88. 